\title{
EFECTO DE TRES FRECUENCIAS DE RIEGO SOBRE LA CALIDAD DE LA FIBRA EN CUATRO CULTIVARES DE ALGODÓN (GOSSYPIUM HIRSUTUM L.) TIPO UPLAND
}

\author{
EFFECT OF THREE IRRIGATION FRECUENCIES ON FIBER QUALITY \\ OF FOUR UPLAND COTTON (GOSSYPIUM HIRSUTUM L.) CULTIVARS
}

\author{
Jesús Rafael Méndez-Natera ${ }^{1}$; Ronald S. Salazar-Brito ${ }^{1}$; \\ José F. Merazo-Pinto ${ }^{1}$; José A. Gil-Marín ${ }^{1}$; Luis Khan-Prado ${ }^{1}$
}

\section{RESUMEN}

Con el objeto de determinar el efecto de diferentes frecuencias de riego (FR) sobre la calidad de la fibra de cuatro cultivares (C) de algodón se realizó un experimento en el Sistema de Riego Santa Elena de las Piñas, Valle del Río Guarapiche, Maturín. Se utilizó un diseño de parcelas divididas, siendo las parcelas principales tres FR (6, 9 y 12 días) y las subparcelas, los cuatro C de algodón (Cabuyare, Deltapine 16, Deltapine 61 y Acala 90-1). La diferencia entre los promedios se detectaron mediante Duncan y el nivel estadístico usado fue de 5\%. El riego se aplicó mediante sifones de 2". Se realizaron dos cosechas, la primera (PC) a los 107 y la segunda (SC) a los 123 días después de la siembra (DDS). No se encontraron diferencias significativas para las FR, ni para los C ni para la interacción FR * C para los siguientes caracteres: contenido de fibra (CF) en la SC (39,80\%), longitud de la fibra (LF) en la PC (1,22 pulg), uniformidad de la fibra en la PC y SC (84,97 y 85,50\%, respectivamente), resistencia de la fibra (RF) en la PC $(25,46 \mathrm{~g} / \mathrm{tex})$. Finura de la fibra (FF) en la SC $(43,33 \mathrm{mic})$, reflectancia de la fibra $(\mathrm{ReF})$ en la SC $(71,26)$ y brillo de la fibra en la PC y SC (9,56 y 9,08, respectivamente). Para el CF en la PC sólo se encontraron diferencias significativas para C, Deltapine 61 tuvo un mayor CF que los C Acala 90-1 y Cabuyare. Para la RF en la SC, las fibras más resistentes se obtuvieron con riegos cada 9 días. La FF en la PC fue mayor en Deltapine 61 y Acala 90-1 en comparación con aquella de Deltapine 16, la ReF fue mayor en los C Acala 90-1 y Deltapine 61 en comparación con Cabuyare. Se encontró una interacción FR * C para la LF en la SC: En la FR de 6 días, las fibras más cortas fueron para Deltapine 16 (1,18 pulg) y en la FR de 12 días fueron para Acala 90-1 (1,08 pulg), mientras que en la FR de 9 días, los cuatro C tuvieron similares LF; por otra parte, en Cabuyare y Deltapine 61, las FR no afectaron la LF, mientras que en Deltapine 16, las fibras más largas se produjeron con riegos cada 12 días, pero para Acala 90-1, este tratamiento produjo las fibras más cortas. En general, las diferentes FR no afectaron marcadamente las propiedades de la fibra a excepción de la longitud de la fibra y la resistencia de la fibra, siendo estos dos parámetros los más importantes para determinar la calidad de la fibra, de allí que el riego debe tenerse como una práctica agronómica de importancia para determinar la calidad de la fibra de algodón.

Palabras clave: Algodón, Gossypium hirsutum, frecuencia de riego, calidad de fibra.

\begin{abstract}
In order to determine the effect of different irrigation frequencies (IF) on fiber quality of four cotton cultivars (CC) an experiment was carried out at Santa Elena de las Piñas Irrigation System, Valley of the Guarapiche River, in Maturin, Venezuela. A split-plot design was used, with three replications, main plots were the three IF (6, 9 and 12 days) and subplots were four CC (Cabuyare, Deltapine 16, Deltapine 61 and Acala 90-1). Means were tested by Duncan multiple range test. The statistical level used was 5\%. Irrigation was applied by means of siphons of 2". Two harvests were carried out, the first harvest (FH) at 107 day after sowing $(D A S)$ and the second harvest $(S H)$ at 123 days. There were not significant differences for $I F, C C$ and $I F * C C$ interaction for the following characters: fiber content $(F C)$ in the $S H(39.80 \%)$, fiber length $(F L)$ in the $F H(1.22$ inch), fiber uniformity in the FH and SH (84.97 and $85.50 \%$, respectively), fiber strength (FS) in the FH (25.46 g/tex). Fiber fineness (FF) in the SH (43.33 micronaire), fiber reflectance (FR) in the SH (71.26) and fiber brightness in the FH and SH (9.56 and 9.08, respectively). For the FC in the FH there were only significant differences for CC, Deltapine 61 had a bigger FC than that Acala 90-1 and Cabu-
\end{abstract}

1 Escuela de Ingeniería Agronómica. Núcleo Monagas. Universidad de Oriente. Avenida Universidad. Campus Los Guaritos. Maturín, 6201. Monagas, Venezuela. Teléfono: 58-91-521192. Fax: 58-91-411029. E-mail: jmendezn@ cantv.net; jalexgil@cantv.net 
yare. For the FS in the SH, the most resistant fibers were obtained with irrigations every 9 days. The FF in the FH was bigger in Deltapine 61 and Acala 90-1 in comparison with that of Deltapine 16, the FR was bigger in CC Acala 90-1 and Deltapine 61 in comparison with that of Cabuyare. There was a significant interaction FR *C for the FL in the SH: In the 6-day IF, the shorter fibers belonged to CC Deltapine 16 (1.18 inch) and in the 12-day IF, belonged to Acala 90-1 (1.08 inch), while in the 9-day IF, the four CC had similar FL. On the other hand, in Cabuyare and Deltapine 61, the IF didn't affect the FL, while in Deltapine 16, the longer fibers occurred with irrigation every 12 days, but for Acala 90-1, this treatment produced the shorter fibers. In general, the different IF didn't affect the fiber properties markedly except for FL and FS, being these two characters, the most important ones to determine the fiber quality, for this reason, cotton irrigation must be a very important agronomic practice in order to achieve a good cotton fiber quality.

Key words: Cotton, Gossypium hirsutum, Irrigation Frequency, Fiber Quality.

\section{INTRODUCCIÓN}

El principal producto del algodón es la fibra que recubre a la semilla. Esta fibra se utiliza en la industria textil para la confección de ropas. Por esta razón la fibra debe poseer una buena calidad. Las propiedades o características relacionadas con la calidad de la fibra son: longitud, resistencia, uniformidad, finura, reflectancia y el brillo de la fibra. De todas éstas, las dos primeras son las más importantes para clasificar a una fibra de buena o regular calidad.

Las propiedades más importantes de la fibra (longitud, resistencia y finura) están fuertemente controladas por factores genéticos y usualmente sólo nominalmente por las condiciones ambientales o las prácticas de manejo. Como resultado, los nombres de las variedades han llegado a ser casi sinónimos con la fibra de una calidad específica. Sin embargo, el ambiente, particularmente la humedad del suelo y la temperatura, pueden contribuir substancialmente a las variaciones en la longitud, resistencia y finura y demás caracteres de la fibra, ya sea directa como indirectamente.

Aunque algunos investigadores en el pasado (Brown et al., 1955; Hamilton et al., 1956; Neely 1940) fueron incapaces de encontrar un cambio apreciable en la fibra, de manera que pudiera ser atribuido a las diferencias de humedad del suelo. Existe una evidencia considerable que una humedad extensa durante al menos la primera mitad del periodo de desarrollo de la bellota puede incrementar la longitud de la fibra en alguna extensión (Bloodworth et al., 1950; Eaton, 1955; Law et al., 1953; Longenecker et al., 1963), los incrementos observados generalmente fueron del orden 1/32 a 1/16 pulgadas, no grandes, pero usualmente lo suficientemente buenos para ser de alguna importancia.

Longenecker et al. (1963) trabajaron con el cultivar de algodón Acala e indicaron que las propiedades de la fibra (longitud, resistencia, finura) fueron sólo afectadas de forma ligera directamente por la frecuencia de riego o el nivel de fertilidad, pero fueron de manera adversa afectadas indirectamente por la fructificación prolongada y el retraso en la maduración atribuido al riego frecuente y al alto contenido de nitrógeno del suelo.

Más recientemente, Hutchinson et al. (1984) trabajaron con 20 cultivares de algodón en suelos de Louisiana, Estados Unidos, con riego y sin riego y encontraron en los tratamientos bajo riego un incremento de la longitud y la resistencia de la fibra, mientras que en los tratamientos sin riego se obtuvieron mayores valores de índice micronaire (finura). Hutchinson et al. (1985), en un estudio realizado en Louisiana, Estados Unidos, demostraron que con el riego en una tasa de 6,4 pulgadas/año durante el periodo 1971-1983, la longitud de la fibra se incrementó de 1,08 a 1,13 pulgadas, representando un aumento del $15 \%$ y aproximadamente un $5 \%$ para la resistencia de la fibra, pero no encontraron diferencias significativas para el porcentaje de fibra ni para el índice micronaire. Gomaa et al. (1981) trabajaron en Egipto con el cultivar Giza 75 durante dos años con aplicaciones de 0, 50, 100, 140 y 190 $\mathrm{kg}$ de nitrógeno/ha e intervalos de riego de 14, 21 y 28 días y encontraron que a mayor frecuencia de riego se logró un aumento en la longitud de la fibra, mientras que el porcentaje de fibra, la finura y la maduración de la fibra fueron mayores en los intervalos de aplicación de riego menores.

Estos reportes indican que no hay una respuesta clara de las propiedades de la fibra (resistencia, longitud, finura, etc.) a la aplicación del riego. De allí que deben realizarse experimentos que conlleven a la obtención de frecuencias de riego óptimas de manera de obtener la mejor calidad de la fibra. El objetivo del presente trabajo fue determinar el efecto de intervalos de riego de 6, 9 y 12 días sobre las propiedades de la fibra (calidad de la fibra) en cuatro cultivares de algodón: 'Cabuyare', 'Deltapine 61', 'Deltapine 16' y 'Acala 90-1'. 


\section{MATERIALES Y MÉTODOS}

El presente trabajo se realizó en el Sistema de Riego del Río Guarapiche, Sector Las Piñas ubicado a $2 \mathrm{~km}$ de la ciudad de Maturín, Edo. Monagas localizado a $9^{\circ} 45^{\prime} 36^{\prime \prime}$ Latitud Norte y $63^{\circ} 11^{\prime} 23^{\prime \prime}$ Longitud Oeste y a 40 m.s.n.m. Se utilizaron los cultivares de algodón 'Cabuyare', 'Deltapine 61', 'Deltapine 16' y 'Acala 90-1'. La distancia entre plantas fue de $0,20 \mathrm{~m}$ y entre hileras de $0,70 \mathrm{~m}$, con tres hileras de $5 \mathrm{~m}$ por tratamiento. El diseño estadístico utilizado fue el de parcelas divididas con tres repeticiones, siendo las parcelas principales las frecuencias de riego de 6, 9 y 12 días y las subparcelas los cultivares de algodón. La siembra se realizó el 9 de marzo para todos los cultivares, el número de días a $50 \%$ de floración ocurrió el 3 de mayo para los cuatro cultivares y la primera cosecha se realizó el 24 de junio y la segunda cosecha el 10 de julio para todos los cultivares. El cuadro 1 muestra el análisis de las características químicas y físicas del suelo donde se llevó a cabo el experimento, realizado en el Laboratorio de Análisis de Suelos y Aguas (LABSAS) de la Universidad de Oriente en Maturín, Edo. Monagas.

La preparación del terreno consistió en un pase de arado, tres pases de rastra y un pase de surcado. La fertilización se realizó en bandas enterradas a razón de $600 \mathrm{~kg}$ de 12-24-12 CP/ha, cinco días después de la siembra y el reabono se realizó en bandas enterradas a razón de $150 \mathrm{~kg}$ de urea/ha a los 40 días después de la siembra. El control de malezas se realizó en forma preemergente a las malezas y al cultivo con Dual a razón de 2 1/ha. Además se practicaron dos limpias manuales una a los 45 días y la otra a los 75 días después de la siembra.

El coeficiente $\mathrm{K}$ del cultivo se determinó mediante la ecuación $\mathrm{K}=\mathrm{ETA} / \mathrm{ETP}$, donde ETA: evapotranspiración real y ETP: evapotranspiración potencial. La ETA se calculó mediante la fórmula $\mathrm{ETA}=\mathrm{dc} / \mathrm{Hf}$, donde dc: láminas consumidas y Hf: número de días entre un muestreo y otro (antes del riego y 48 horas después del riego anterior). La dc se calculó mediante la fórmula dc $=(\mathrm{Hf} 2-\mathrm{Hf} 1) / 100$

Cuadro 1

Algunas características químicas y físicas donde se realizó el experimento con cuatro cultivares de algodón probados en parcelas del Sistema de Riego "Santa Elena de las Piñas”, del Río Guarapiche, Maturín, Edo. Monagas.

\begin{tabular}{|l|c|c|c|l|}
\hline \multirow{2}{*}{ Características } & \multicolumn{3}{|c|}{ Estratos (cm) } & \multicolumn{2}{c|}{ Metodología 1/ } \\
\cline { 2 - 4 } & $\mathbf{0 - 2 0}$ & $\mathbf{2 0 - 4 0}$ & $\mathbf{4 0 - 6 0}$ & \\
\hline $\mathrm{pH}$ & 5,5 & 5,7 & 5,9 & Potenciómetro (1:1) \\
\hline Materia Orgánica (\%) & 1,56 & 1,12 & 0,78 & Walkley-Black, colorímetro \\
\hline P (ppm) & 51,8 & 39,9 & 30,0 & Bray N 1 1, Azul, Mol Ac. Asc. \\
\hline Al (meq/100 g suelo) & - & - & - & KCl, 1 N Titulación \\
\hline Ca (meq/100 g suelo) & 6,52 & 5,69 & 4,95 & KCl, 1 N. Absorc. Atómica \\
\hline Mg (meq/100 g suelo) & 1,18 & 1,03 & 0,70 & KCl, 1 N. Absorc. Atómica \\
\hline K (meq/100 g suelo) & 0,03 & 0,01 & 0,01 & Bray N 1, Absorc. Atómica \\
\hline CICE (meq/100 g suelo) & 7,73 & 6,73 & 5,66 & K de Cationes (Ca, Al, Mg, K) \\
\hline Saturación de AL (\%) & - & - & - & (Al/CICE)*100 \\
\hline Saturación de Ca (\%) & 84,35 & 84,55 & 87,45 & (Ca/CICE)*100 \\
\hline Saturación de Mg (\%) & 15,26 & 15,30 & 12,37 & (Mg/CICE)*100 \\
\hline Saturación de K (\%) & 0,39 & 0,15 & 0,18 & (K/CICE)*100 \\
\hline Textura & FAa & FAa & FAa & Bouyoucos \\
\hline Contenido de arcilla (\%) & 23,2 & 21,2 & 21,2 & Bouyoucos \\
\hline
\end{tabular}

1/ Realizado en el Laboratorio de Análisis de Suelos y Aguas (LABSAS) de la Universidad de Oriente en Maturín, Edo. Monagas. 
* Da * Pr, donde Hf2: Contenido de humedad 48 horas después del riego anterior; Hf1: contenido de humedad antes del riego; Da: densidad aparente del estrato y Pr: profundidad radical en cada estrato. La humedad del suelo se determinó por el método gravimétrico, recolectando muestras sucesivas antes del riego y 48 horas después de éste. La humedad del suelo se calculó en cada frecuencia de riego para cada repetición, es decir, tres repeticiones por frecuencia de riego. El Hf se calculó mediante la fórmula $\mathrm{Hf}=[(\mathrm{PSH}-\mathrm{PSS}) / \mathrm{PSS}] * 100$, donde PSH: Peso (g) de suelo húmedo, PSS: Peso (g) de suelo seco. Los estratos estuvieron comprendidos entre 0-20 cm, 20-40 cm y 40-60 cm de profundidad. La ETP se calculó promediando los métodos de García y López, Thorhwaite y Blaney-Criddle (cuadro 2). El cuadro 3 muestra las temperaturas medias $\left({ }^{\circ} \mathrm{C}\right)$, la precipitación $(\mathrm{mm})$ y la humedad relativa $(\%)$ registradas durante el desarrollo del ensayo.

\section{Cuadro 2}

Evapotranspiración potencial ( $\mathrm{mm} /$ día) promedio mensual correspondiente al periodo de riego con cuatro cultivares de algodón probados en parcelas del Sistema de Riego “Santa Elena de las Piñas”, del Río Guarapiche, Maturín, Edo. Monagas

\begin{tabular}{|c|c|c|c|c|}
\hline \multirow{2}{*}{ Mes } & \multicolumn{3}{|c|}{ Métodos } & \multirow{2}{*}{ Promedio } \\
\cline { 2 - 4 } & Thorhwaite & $\begin{array}{c}\text { García y } \\
\text { López }\end{array}$ & $\begin{array}{c}\text { Blaney- } \\
\text { Criddle }\end{array}$ & \\
\hline Abril & 4,75 & 5,95 & 4,35 & 5,02 \\
\hline Mayo & 4,92 & 5,80 & 3,53 & 4,75 \\
\hline Total & 9,67 & 11,45 & 7,88 & 9,77 \\
\hline
\end{tabular}

Se realizaron cinco riegos de asiento (hasta los 19 días después de la siembra), de manera de establecer la población de los diferentes cultivares. Luego se aplicaron las frecuencias de riego (20 días después de la siembra). La lámina de riego promedio aplicada fue de $366 \mathrm{~mm}$, contando a partir del establecimiento del ensayo y en los cinco riegos de establecimiento se aplicaron $80 \mathrm{~mm}$ de agua aproximadamente, para un total de $440 \mathrm{~mm}$ de agua. En cada riego se aplicó una lámina promedio de 6 $\mathrm{mm}$ /día, tomando en consideración las condiciones de la zona. Como fueron tres frecuencias de riego, por lo tanto, las láminas aplicadas $(\mathrm{Dn}=\mathrm{FR} * \mathrm{ETP}$, donde $\mathrm{Dn}=$ Lámina neta aplicada, $\mathrm{Fr}=$ Frecuencia de riego y ETP = Evapotranspiración potencial) fueron en cada caso. Para la frecuencia de 6 días: $\mathrm{Dn}=6 * 6 \mathrm{~mm}=36 \mathrm{~mm}$; para la frecuencia de 9 días: $\mathrm{Dn}=9 * 6 \mathrm{~mm}=54 \mathrm{~mm}$ y para la frecuencia de 12 días: $\mathrm{Dn}=12 * 6 \mathrm{~mm}=72 \mathrm{~mm}$. El riego se realizó mediante sifones de 2 " y las cargas hidráulicas de trabajo de las mismas fueron calibradas a nivel de campo con un Ballofet, como se conoce el caudal $\mathrm{Q}$ y como $\mathrm{Q}=\mathrm{V} / \mathrm{T}$, donde $\mathrm{V}=$ Volumen de agua aplicada y $\mathrm{T}=$ Tiempo de riego; el $\mathrm{V}$ se puede calcular mediante la ecuación: $\mathrm{V}=\mathrm{A} * \mathrm{Dn}$, de allí que $\mathrm{T}=\mathrm{V} / \mathrm{Q}$, obteniéndose así el tiempo de aplicación para cada frecuencia.

Para este experimento se determinaron las siguientes características de la fibra: contenido de fibra, longitud de la fibra, uniformidad de la fibra, resistencia de la fibra, finura de la fibra, reflectancia de la fibra y brillo de la fibra. Todas estas características de la fibra se determinaron en dos cosechas: la primera a los 107 días y la segunda a los 123 días después de la siembra. Se realizó el análisis de varianza convencional y las diferencias entre tratamientos se detectaron mediante la prueba de rangos múltiples de Duncan. El nivel de significación fue $5 \%$.

\section{RESULTADOS Y DISCUSIÓN}

\section{Primera Cosecha a los 107 días después de la siembra}

No se encontraron diferencias significativas para las frecuencias de riego (FR), ni para los cultivares (C) ni para la interacción $\mathrm{FR} * \mathrm{C}$ para los siguientes caracteres: longitud de la fibra (1,22 pulgadas), uniformidad de la fibra $(84,97 \%)$, resistencia de la fibra $(25,46 \mathrm{~g} / \mathrm{tex})$ y brillo de la fibra $(9,56)$.

Las bellotas con un mayor contenido de fibra correspondieron a las del cultivar Deltapine 61, mientras el menor contenido de fibra fue registrado por los cultivares Acala 90-1 y Cabuyare (Figura 1). La Figura 2 muestra la prueba de Duncan para la finura de la fibra (índice micronaire). Las fibras más gruesas correspondieron a aquellas de los cultivares Deltapine 61 y Acala 90-1, mientras el cultivar Deltapine 16 presentó las fibras más finas. La Figura 3 muestra la reflectancia de la fibra, los cultivares con fibras más reflectantes fueron Acala 90-1 y Deltapine 61, superando a la reflectancia de las fibras del cultivar Cabuyare. 


\section{Cuadro 3}

Temperaturas medias $\left({ }^{\circ} \mathrm{C}\right)$, precipitación $(\mathrm{mm})$ y humedad relativa $(\%)$ registradas en el experimento con cuatro cultivares de algodón probados en parcelas del Sistema de Riego "Santa Elena de las Piñas", del Río Guarapiche, Maturín, Edo. Monagas

\begin{tabular}{|c|c|c|c|c|c|c|c|c|c|c|c|c|c|c|c|}
\hline \multirow{2}{*}{ Días } & \multicolumn{3}{|c|}{ Marzo } & \multicolumn{3}{|c|}{ Abril } & \multicolumn{3}{|c|}{ Mayo } & \multicolumn{3}{|c|}{ Junio } & \multicolumn{3}{|c|}{ Julio } \\
\hline & TM & $\mathbf{P}$ & HR & $\mathbf{T M}$ & $\mathbf{P}$ & HR & $\mathbf{T M}$ & $\mathbf{P}$ & HR & TM & $\mathbf{P}$ & HR & TM & $\mathbf{P}$ & HR \\
\hline 1 & & & & 26,6 & - & 61 & 27,9 & - & 59 & 26,7 & - & 75 & 26,0 & 4,1 & 86 \\
\hline 2 & & & & 26,5 & - & 55 & 26,7 & - & 71 & 25,2 & 23,0 & 83 & 25,3 & 6,6 & 91 \\
\hline 3 & & & & 26,7 & - & 55 & 27,6 & - & 66 & 23,8 & 34,8 & 90 & 25,4 & 11,4 & 84 \\
\hline 4 & & & & 26,1 & - & 60 & 28,0 & - & 61 & 24,8 & 16,3 & 80 & 25,7 & 0,8 & 81 \\
\hline 5 & & & & 24,6 & - & 74 & 27,6 & - & 61 & 25,0 & 0,3 & 82 & 25,9 & 1,9 & 82 \\
\hline 6 & & & & 25,8 & - & 59 & 27,2 & - & 59 & 25,0 & 28,5 & 88 & 25,4 & 8,3 & 90 \\
\hline 7 & & & & 26,0 & - & 57 & 27,5 & - & 60 & 26,1 & - & 79 & 26,7 & - & 78 \\
\hline 8 & & & & 26,7 & - & 64 & 26,8 & - & 61 & 25,0 & 43,3 & 87 & 24,9 & 2,6 & 79 \\
\hline 9 & 25,6 & - & 63 & 26,8 & - & 63 & 27,3 & - & 60 & 27,2 & 1,4 & 75 & 27,0 & 17,8 & 93 \\
\hline 10 & 26,5 & - & 65 & 26,5 & - & 61 & 27,0 & - & 68 & 27,0 & 9,1 & 79 & 27,4 & - & 79 \\
\hline 11 & 26,5 & - & 64 & 26,9 & - & 60 & 27,8 & - & 59 & 27,4 & 7,9 & 75 & & & \\
\hline 12 & 26,2 & - & 65 & 27,8 & - & 60 & 26,5 & - & 60 & 27,4 & 4,1 & 77 & & & \\
\hline 13 & 25,9 & - & 58 & 27,4 & - & 54 & 27,4 & - & 64 & 27,7 & - & 72 & & & \\
\hline 14 & 25,5 & - & 64 & 26,9 & - & 62 & 27,3 & - & 65 & 28,1 & - & 69 & & & \\
\hline 15 & 26,9 & - & 55 & 27,4 & - & 62 & 27,9 & - & 65 & 26,1 & 3,4 & 83 & & & \\
\hline 16 & 26,7 & - & 55 & 27,6 & - & 65 & 27,0 & - & 67 & 25,6 & 10,0 & 90 & & & \\
\hline 17 & 26,8 & - & 62 & 27,6 & - & 64 & 27,7 & - & 60 & 24,9 & 13,3 & 92 & & & \\
\hline 18 & 26,9 & 0,3 & 63 & 27,1 & - & 62 & 27,3 & - & 67 & 25,6 & 3,1 & 92 & & & \\
\hline 19 & 26,8 & - & 68 & 27,4 & - & 61 & 26,2 & - & 64 & 25,1 & 12,0 & 92 & & & \\
\hline 20 & 27,5 & - & 69 & 27,1 & - & 66 & 27,6 & - & 63 & 25,7 & 10,0 & 85 & & & \\
\hline 21 & 27,9 & - & 69 & 26,9 & - & 68 & 27,8 & - & 62 & 27,2 & 0,1 & 77 & & & \\
\hline 22 & 27,0 & - & 68 & 26,6 & - & 69 & 27,8 & - & 65 & 25,8 & 0,8 & 88 & & & \\
\hline 23 & 27,5 & - & 63 & 26,9 & - & 67 & 28,1 & - & 67 & 27,4 & 0,4 & 76 & & & \\
\hline 24 & 27,6 & - & 59 & 27,3 & - & 62 & 27,1 & - & 66 & 27,4 & - & 74 & & & \\
\hline 25 & 27,3 & - & 63 & 27,4 & - & 62 & 27,4 & - & 65 & 27,9 & - & 69 & & & \\
\hline 26 & 27,2 & - & 59 & 27,3 & - & 61 & 27,5 & - & 66 & 27,4 & 0,5 & 72 & & & \\
\hline 27 & 26,8 & - & 60 & 27,6 & - & 61 & 28,2 & - & 61 & 27,1 & 1,0 & 77 & & & \\
\hline 28 & 26,6 & - & 66 & 27,5 & - & 64 & 25,0 & 7,8 & 83 & 26,2 & 0,2 & 77 & & & \\
\hline 29 & 26,9 & - & 61 & 27,9 & - & 62 & 24,0 & 0,5 & 89 & 25,5 & 0,4 & 90 & & & \\
\hline 30 & 27,4 & - & 61 & 27,7 & - & 60 & 26,4 & 2,5 & 77 & 25,4 & 6,8 & 80 & & & \\
\hline 31 & 26,9 & - & 58 & & & & 25,5 & - & 84 & & & & & & \\
\hline Total & 616,9 & 0,3 & 1418 & 808,6 & 0,0 & 1961 & 841,1 & 10,8 & 2045 & 786,7 & 230,7 & 2425 & 259,7 & 53,5 & 843 \\
\hline Prom & 26,8 & & 61,6 & 27,0 & & 62,0 & 27,1 & & 66,0 & 26,2 & & 80,8 & 26,0 & & 84,3 \\
\hline $\mathrm{ADCC}$ & 616,9 & 0,3 & & 1425,5 & 0,3 & & 2266,6 & 11,1 & & 3053,3 & 241,8 & & 3313,0 & 295,3 & \\
\hline
\end{tabular}

TM: Temperatura Media $\left({ }^{\circ} \mathrm{C}\right)$ P: Precipitación (mm) HR: Humedad Relativa (\%)

ADCC: Acumulado durante el ciclo del cultivo

Fuente : Estación Meteorológica de la Fuerza Aérea Venezolana, Maturín, Edo. Monagas 


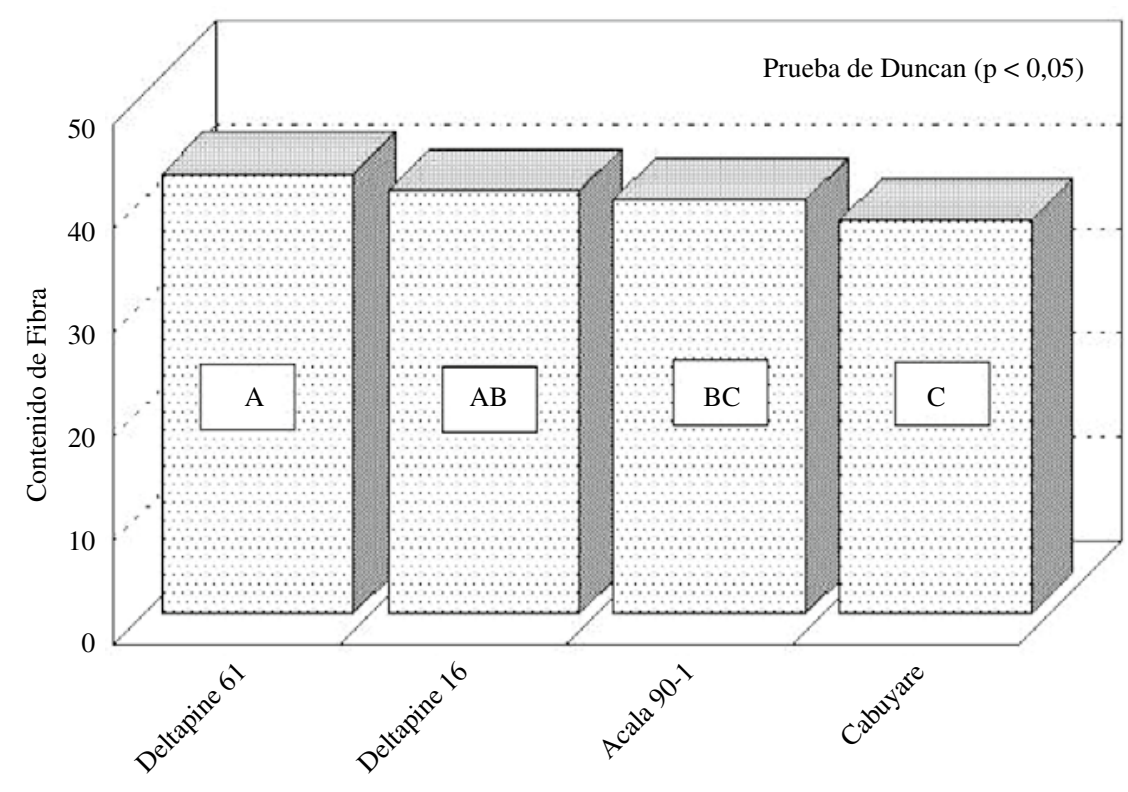

Cultivares

Figura 1. Promedios del contenido de fibra en la primera cosecha de cuatro cultivares de algodón. Letras iguales indican promedios estadísticamente iguales.

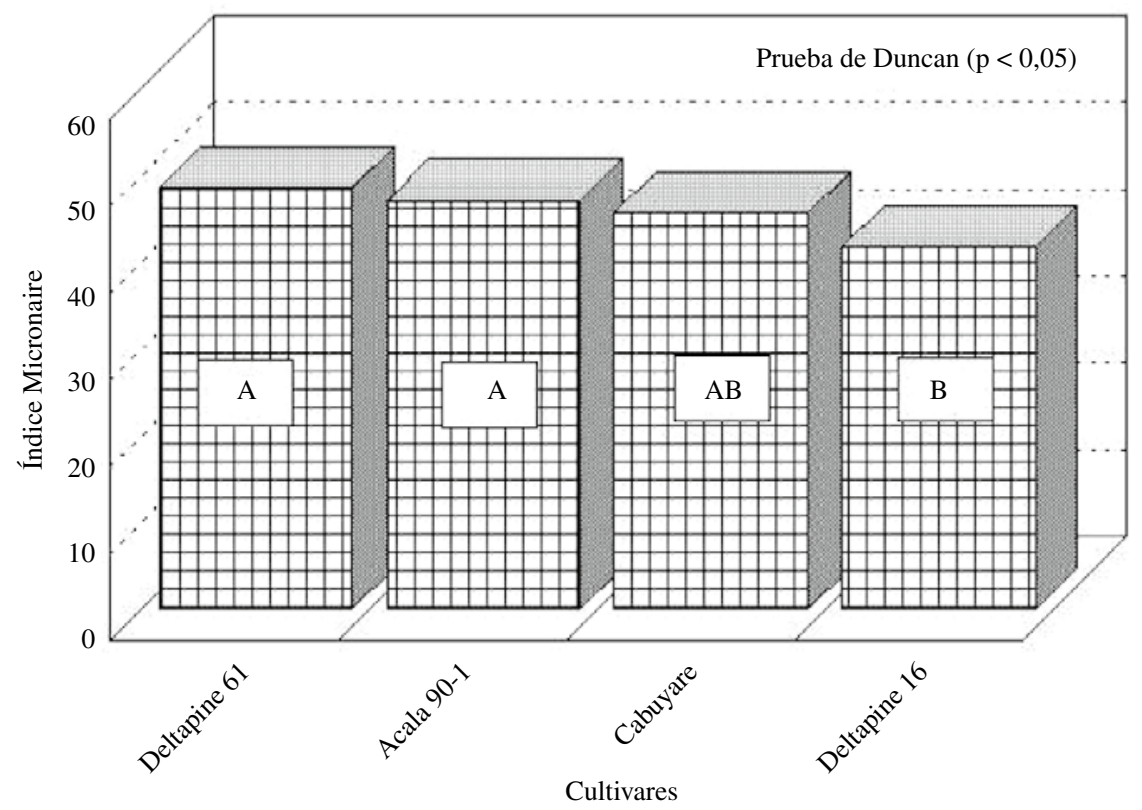

Figura 2. Promedios de la finura de la fibra (índice micronaire) en la primera cosecha de cuatro cultivares de algodón. Letras iguales indican promedios estadísticamente iguales. 


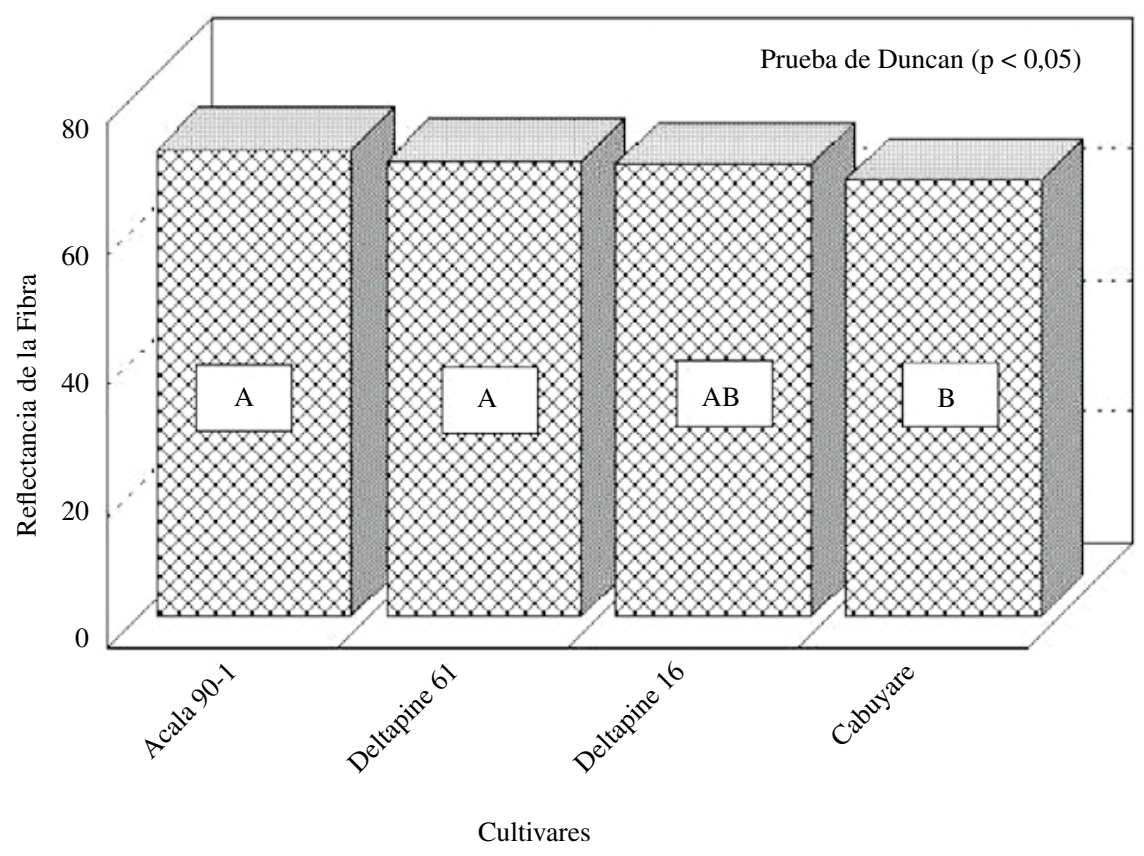

Figura 3. Promedios de la reflectancia de la fibra en la primera cosecha de cuatro cultivares de algodón. Letras iguales indican promedios estadísticamente iguales.

\section{Segunda Cosecha a los 123 días después de la siembra}

No se encontraron diferencias significativas para las frecuencias de riego (FR) ni para los cultivares (C) ni para la interacción FR * C para los siguientes caracteres: contenido de fibra $(39,80 \%)$, uniformidad de la fibra $(85,50 \%)$, finura de la fibra $(43,33$ micronaire), reflectancia de la fibra $(71,26)$ y brillo de la fibra $(9,08)$. El cuadro 4 muestra la prueba de promedios de Duncan para la interacción significativa de los cultivares $*$ las frecuencias de riego para la longitud de la fibra. En la frecuencia de riego de 6 días, las fibras más cortas fueron para Deltapine 16 $(1,18$ pulg) y en la frecuencia de riego de 12 días fueron para Acala 90-1 (1,08 pulg), mientras que en la frecuencia de riego de 9 días, los cuatro cultivares tuvieron similares longitudes de fibra. Por otra parte, en Cabuyare y Deltapine 61, las frecuencias de riego no afectaron la longitud de la fibra, mientras que en Deltapine 16 las fibras más largas se produjeron con riegos cada 12 días, pero para Acala 90-1 este tratamiento produjo las fibras más cortas. La Figura 4 muestra la prueba de Duncan para la resistencia de la fibra bajo tres frecuencias de riego. Las fibras más resistentes se obtuvieron con riegos cada 9 días sin importar el cultivar.

\section{Cuadro 4}

Promedios de la longitud de la fibra en la segunda cosecha de cuatro cultivares de algodón sometidos a tres frecuencias de riego

\begin{tabular}{|l|c|c|c|c|c|c|}
\hline \multirow{2}{*}{ Cultivares } & \multicolumn{5}{|c|}{ Longitud de la fibra 1/ } \\
\cline { 2 - 7 } & \multicolumn{5}{|c|}{ Frecuencia de Riego (días) } \\
\cline { 2 - 7 } & \multicolumn{2}{|c|}{6} & \multicolumn{2}{c|}{9} & \multicolumn{2}{c|}{12} \\
\hline Cabuyare & 1,28 & $\mathrm{Aa}$ & 1,22 & $\mathrm{Aa}$ & 1,21 & $\mathrm{Aa}$ \\
\hline Deltapine 61 & 1,29 & $\mathrm{Aa}$ & 1,24 & $\mathrm{Aa}$ & 1,22 & $\mathrm{Aa}$ \\
\hline Deltapine 16 & 1,18 & $\mathrm{Bb}$ & 1,19 & $\mathrm{Ab}$ & 1,30 & $\mathrm{Aa}$ \\
\hline Acala $90-1$ & 1,21 & $\mathrm{ABa}$ & 1,22 & $\mathrm{Aa}$ & 1,08 & $\mathrm{Bb}$ \\
\hline
\end{tabular}

1/ Prueba de rangos múltiples de Duncan ( $<<0,05)$. Letras iguales indican promedios estadísticamente iguales. Letras mayúsculas para las comparaciones verticales Letras minúsculas para las comparaciones horizontales

En general, las frecuencias de riego de 6, 9 y 12 días no afectaron marcadamente la calidad de la fibra a excepción de la longitud y resistencia de la fibra. Esto se debió probablemente a que las propiedades de la fibra tienen un mayor componente genético, es decir, dependen de los genotipos envueltos más que de las prácticas culturales aplicadas. Es de 


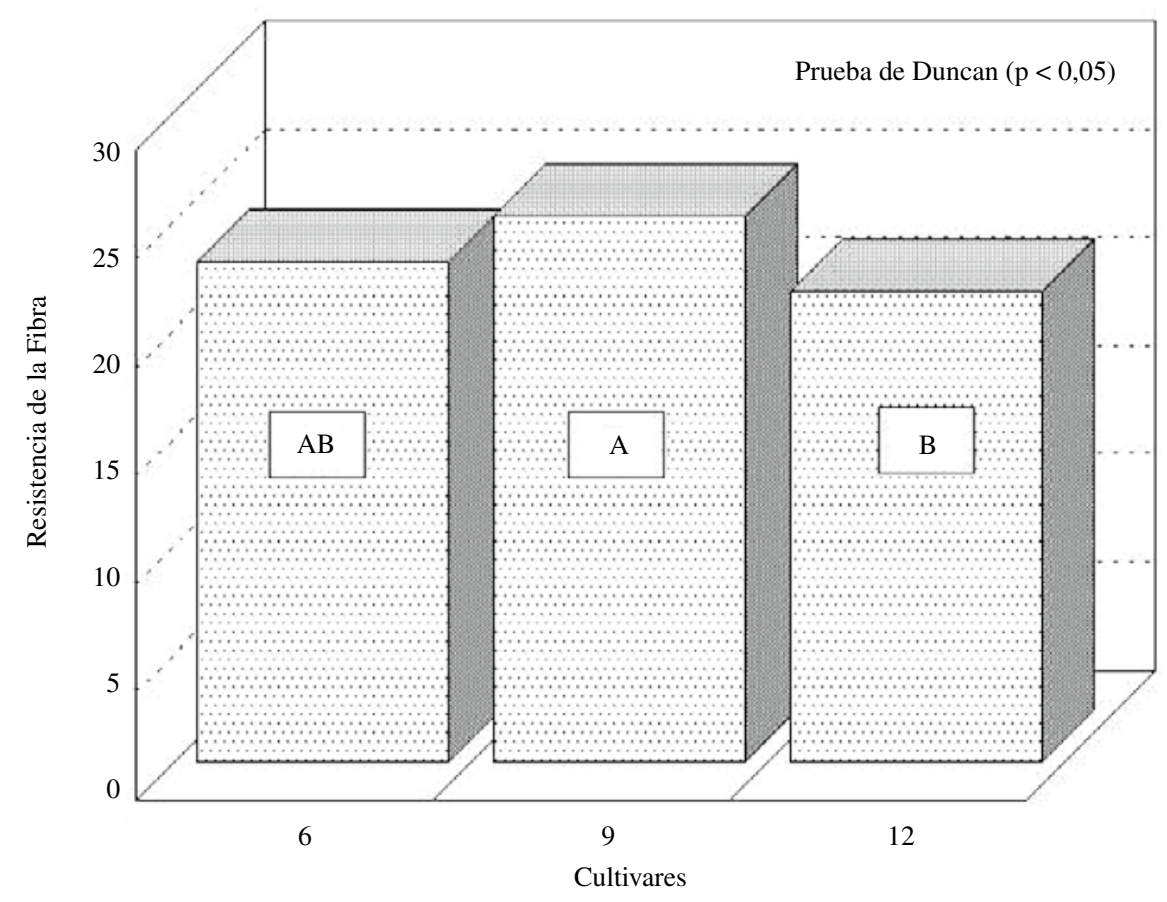

Figura 4. Promedios de la resistencia de la fibra en la segunda cosecha en el cultivo de algodón sometido a tres frecuencias de riego. Letras iguales indican promedios estadísticamente iguales.

hacer notar que las menores frecuencias de riego incrementaron la resistencia de la fibra. Resultados similares fueron reportados por Hutchinson et al. $(1984,1985)$. Resultados diferentes fueron indicados por Gomaa et al. (1981), quienes utilizaron al cultivar Giza 75 durante dos años con intervalos de riego de 14, 21 y 28 días y encontraron que el porcentaje de fibra, la finura y la maduración de la fibra fueron mayores en los intervalos de aplicación de riego menores.

Por otra parte, para la longitud de la fibra, los resultados de este experimento fueron interesantes. Mientras las frecuencias de riego no afectaron la longitud de la fibra de los cultivares Cabuyare y Deltapine 61, sí afectaron la de los otros dos cultivares, pero de manera contraria, en Acala 90-1, la menor longitud se obtuvo con la frecuencia de riego de 12 días. $\mathrm{Al}$ respecto Bradow y Davidonis (2000) indicaron que déficit severos de humedad durante la etapa de elongación de la fibra reducen la longitud de la fibra, en apariencia debido simplemente a los procesos fisiológicos y mecánicos de la expansión celular, pero en Deltapine 16, este tratamiento fue el que produjo la mayor longitud de la fibra; este último resultado fue similar al reportado por Gomaa et al. (1981), quienes indicaron que a mayor frecuencia de riego se logró un aumento en la longitud de la fibra. A la luz de estos resultados, se observa que la respuesta de las propiedades de la fibra y en especial la longitud de la fibra no sigue un patrón definido y que es el cultivar utilizado el que aportará las mayores diferencias en cuanto a este carácter. Al respecto de estos resultados contrastantes en relación a la respuesta de los cultivares a la aplicación de diferentes frecuencias de riego, Bradow y Davidonis (2000) indicaron que la disponibilidad de agua y la duración y tiempo de floración y producción de bellotas puede resultar en interacciones fisiológicas complejas entre los déficits de humedad y las propiedades de la fibra incluyendo la longitud.

En este ensayo, las frecuencias de riego sólo afectaron la longitud y resistencia de la fibra. Pettigrew (2001) indicó que los déficits de humedad reducen la longitud de la fibra, pero el estrés hídrico necesita ser severo y ocurrir brevemente después de la floración para producir una reducción significativa de la longitud de la fibra y que ese estrés por sequía puede también reducir la finura (micronaire) de la fibra, pero que este efecto se deba probablemente 
a las reducciones en la capacidad fotosintética del dosel. Palomo-Gil (2004) evaluaron durante tres años consecutivos (1997 a 1999) tres tratamientos de riego consistentes en dos, tres y cuatro riegos de auxilio, aplicándose el último a 80, 100 y 120 días después de la siembra (dds), respectivamente y seis dosis de nitrógeno $(0,40,80,120,160$ y 200 $\mathrm{kg} / \mathrm{ha}$ ) para evaluar el rendimiento de algodón en rama y de fibra, los componentes del rendimiento y la calidad de la fibra (longitud, resistencia y finura) y encontraron que todas las variables estudiadas resultaron afectadas por el año, número de riegos y dosis de nitrógeno pero no se detectó ningún tipo de interacción. Con tres y cuatro riegos de auxilio se observó una mejor calidad de fibra que con dos riegos.

Finalmente, en este ensayo no se encontraron diferencias ni para los cultivares ni las frecuencias de riego y su interacción en ambas épocas de cosecha para la uniformidad de la fibra y brillo de la fibra indicando poca variación para estos dos caracteres, mientras que para el resto de los caracteres (contenido de fibra, longitud, resistencia, finura y reflectancia de la fibra) tampoco se encontraron diferencias significativas para ninguna de las fuentes de variación

\section{LITERATURA CITADA}

BLOODWORTH, M. E.; W. R. COWLEY AND J. S. MORRIS. 1950. Growth and yield of cotton on Willacy loam as affected by different irrigation levels. Texas Experimental Station. Progress Report 1217.

BRADOW, J. M. AND DAVIDONIS, G. H. 2000. Quantitation of fiber quality and the cotton production-processing interface: A physiologist's perspective. The Journal of Cotton Science 4: 34-64.

BROWN, D. A., R. H. BENEDICT AND B. B. BRYAN. 1955. Irrigation of cotton in Arkansas. Arkansas Experimental Station. Bulletin 552.

Eaton, F. M. 1955. Physiology of the cotton plant. Annual Review of Plant Physiology 6: 299-328.

GOMAA, M.; K. EL SAYED AND M. RADY. 1981. Response of Egyptian cotton to nitrogen fertilizer and irrigation frequency. II. Fiber properties and seed quality characters. Monoufeia Journal of Agricultural Research 4: 189-210.

HAMILTON, J.; C. O. STANBERRY AND W. M. WOOTTON. 1956. Cotton growth and production as affected by moisture, nitrogen and plant spacing on the Yuma Mesa. Proceedings of the Soil Science Society of America 20: 246-252.

HUTCHINSON, R.; W. CALDWELL; T. TALBOT AND T. SHARPE. 1984. Irrigated and non-irrigated cotton variety and strains tests. Annuals Progress Report. Northeast al menos para alguna de las dos épocas de cosecha, id est, en la primera cosecha no variaron la longitud y resistencia de la fibra y para la segunda cosecha no variaron el contenido de fibra, finura y reflectancia de la fibra, indicando poca variación de la calidad de la fibra ante los tratamientos evaluados: cultivares y frecuencias de riego.

\section{CONCLUSIONES}

Las diferentes frecuencias de riego no afectaron marcadamente las propiedades de la fibra a excepción de la longitud de la fibra y la resistencia de la fibra, siendo estos dos parámetros los más importantes para determinar la calidad de la fibra; de allí que el riego debe tenerse como una práctica agronómica de importancia para determinar la calidad de la fibra de algodón.

\section{AGRADECIMIENTO}

Al Consejo de Investigación de la Universidad de Oriente por el soporte dado al primer autor.
Research Station, St. Joseph, L. A. and Macon Ridge. Louisianna University.

HUTCHINSON, R.; S. PHILLIPS; T. TALBOT AND J. BARTLESON. 1985. Effect of irrigation on cotton production on the loessial soils of the Macon Ridge in Northeast Louisiana. Agronomy Journal 7 (3): 457-460.

LAW, W. P.; C. M. LUND AND J. H. ANDERSON. 1953. Cotton irrigation experiment, 1953. South Carolina Agricultural Eng. Department. Progress Report.

LONGENECKER, D. E.; E. L. THAXTON, JR. AND P. J. LYERLY. 1963. Cotton production in far west Texas with emphasis on irrigation and fertilization. Texas Experimental Station Bulletin 1001.

NEELY, W. J. 1940. The effect of genetical factors, seasonal differences and soil variation upon certain characteristics of upland cotton in the Yazoo-Mississippi Delta. Mississippi Experimental Station Technical Bulletin 28.

PALOMO-GIL, A.; GAYTÁN-MASCORRO, A.; FAZCONTRERAS, R.; RETA-SÁNCHEZ, D. G. Y GUTIÉRREZ DEL RÍO, E. 2004. Rendimiento y calidad de fibra de algodón en respuesta al número de riegos y dosis de nitrógeno. TERRA Latinoamericana 22 (3): 299-305.

PETTIGREW, W. T. 2001. Environmental effects on cotton fiber carbohydrate concentration and quality. Crop Science 41: 1108-1113. 
